so that the responsibility for safe working shall be fixed on those who have the effective powers of control and not solely, as at present, on the technical employees. Among the many technical recommendations relating to mining practice are important proposals for laying down and maintaining more definite standards of ventilation, for improving and intensifying the system of supporting the underground workings and securing the general application of the principles of roof control, which have been the subject of much recent research work, for improving the physical and material conditions under which haulage opera- tions are conducted below ground, and for strengthening the precautions in connexion with explosives, electricity, shot-firing and coal dust.

In conclusion, while doubtless many of the recommendations of the Commissioners will be hotly debated when the new Bill is passing through the House of Commons, its most bitter opponent must acknowledge that the Commissioners have carried out their difficult task with moderation and vision. They have attempted to be helpful to everybody engaged in the production of coal, and appear to have held the scales of equity admirably.
J. A. S. Ritson.

\title{
Liquid Helium
}

By Dr. J. F. Allen, Royal Society Mond Laboratory, and Dr. H. Jones, Imperial College, London

$\mathrm{T}$ HE properties of liquid helium can best be considered under two headings: (a) properties in thermal equilibrium, $(b)$ transport effects. The equilibrium properties, which have been the subject of many careful investigations in Leyden and elsewhere, may be regarded as fairly well established. These include the determination of the specific heat for different temperatures, the variation of density with temperature at different constant pressures, and the relation of the saturated vapour pressure to the absolute temperature scale. The investigations of transport effects such as the flow of the liquid through tubes, heat conductivity and associated effects are still in an early stage of development, and no clear understanding of these phenomena has yet been reached. The subject was discussed at a meeting following the International Refrigeration Congress in July and also during the Cambridge meeting of the British Association. In this article we shall confine our attention largely to the interesting newly discovered transport effects, and give an account of recent experiments the results of which at present seem to find general acceptance.

\section{Equilibrium Properties of Liquid Helium}

Helium at atmospheric pressure liquefies at $4.22^{\circ} \mathrm{K}$.; the critical temperature is $5.2^{\circ} \mathrm{K}$. Generally speaking, the temperature range over which the properties of liquid helium have been measured extends only down to $1^{\circ} \mathrm{K}$., since this is the lowest temperature conveniently reached by lowering the vapour pressure over the surface of the liquid. At $2 \cdot 19^{\circ} \mathrm{K}$. under its own vapour pressure, liquid helium undergoes a remarkable transformation. As the liquid is cooled through $2 \cdot 19^{\circ} \mathrm{K}$., the specific heat jumps suddenly from a value of 0.4 cal. per gm. per degree to more than 5 cal. per gm. per degree, thereafter falling rapidly, approximately as $T^{5}$. Simon has recently shown that at very low temperatures $\left(0.02^{\circ}-0.05^{\circ} \mathrm{K}\right.$.) produced by adiabatic demagnetization of iron ammonium alum, the specific heat of liquid helium varies as $T^{3}$.

The transformation at $2 \cdot 19^{\circ} \mathrm{K}$. has also a remarkable effect on the expansion coefficient of liquid helium. Above that temperature it is positive, while below it is negative, although there is no discontinuity in the value of the density itself at $2 \cdot 19^{\circ} \mathrm{K}$.

Phase transformations of the type that liquid helium undergoes at $2 \cdot 19^{\circ} \mathrm{K}$. are known in other branches of physics; for example, the Curiepoint transformation of a ferromagnetic, the orderdisorder transformation of certain alloys, and the transition between the superconductive and the normal state of a metal. The temperature of the transformation in liquid helium is known as the $\lambda$-point, a name introduced by Ehrenfest. The modification of the liquid below the $\lambda$-point is generally referred to as helium II, that above the $\lambda$-point as helium $\mathrm{I}$.

Liquid helium at $1^{\circ} \mathrm{K}$. can be solidified under an external pressure of 25 atmospheres. At higher temperatures, greater pressures are required to produce the solid. The properties of liquid helium I are such as would be expected of an ordinary liquid of very low boiling point. It is far otherwise with liquid helium II. In the first place, it is an 
immediate inference from the phase diagram that liquid helium II under its own vapour pressure remains liquid at absolute zero, for even in the neighbourhood of $1^{\circ} \mathrm{K}$. the boundary line between the solid and liquid phases tends to become parallel with the temperature axis at a pressure of approximately 25 atmospheres. Since $d p / d t$ (the slope of the boundary line) approaches zero as the temperature is lowered, and since the volume change, $\Delta v$, is not zero, it follows from Clapeyron's equation $d p / d t=\Delta s / \Delta v$, that $\Delta s$, the change in entropy on passing from liquid to solid, also approaches zero. There can thus exist the paradoxical situation of a liquid with zero entropy. On this account the possibility has been considered by several investigators that condensed helium at absolute zero has a space-ordered structure, and that the $\lambda$-point is of the nature of an order-disorder transformation.

An experimental investigation of this point has been carried out by Keesom and Taconis. They examined the reflection of $\mathrm{X}$-rays in a column of liquid helium II at about $1 \cdot 6^{\circ} \mathrm{K}$., as well as in helium above the $\lambda$-point. No essential difference between the reflections in the two cases could be observed. Confirmation of the space-ordered theory, therefore, is still lacking, although it should be borne in mind that at $1.6^{\circ} \mathrm{K}$. an appreciable degree of disorder would in any event be expected.

We may mention here that F. London, in a very interesting letter to NATURE, has recalled a prediction by Einstein that a perfect Bose gas at sufficiently low temperatures should show a discontinuity in the temperature derivative of the specific heat. Applying Einstein's formula to liquid helium, London shows that the discontinuity would occur at $3 \cdot 09^{\circ} \mathrm{K}$. Although Einstein's discontinuity is only in the temperature derivative of the specific heat, not in the specific heat itself, London suggests that there may be some connexion with the $\lambda$-point of liquid helium. The advantage of such a theory of the $\lambda$-point is that it appears not to necessitate a space-ordered structure for the liquid at low temperatures.

\section{TRANSPORT EFFECTS}

Flow Phenomena. Experiments designed to measure the viscosity of liquid helium II have led to the most surprising and apparently contradictory results. The first attempt in this direction was made at Toronto by Burton and Misener, who measured the damping of an oscillating cylinder immersed in the liquid. The value for the viscosity was found to drop suddenly at the $\lambda$-point from approximately $10^{-4}$ c.G.S. units for helium $I$ to $10^{-5}$ c.G.S. units for helium II. Recent and more precise measurements by the similar method of an oscillating disk, made by MacWood at Leyden, give values varying from $3 \times 10^{-5}$ c.G.S. units for helium II just below the $\lambda$-point to $2 \times 10^{-6}$ c.G.S. units at $1 \cdot 1^{\circ} \mathrm{K}$. Liquid helium II thus appears to be very much less effective in damping the motion of a body immersed in it than helium gas at room temperature.

Attempts to measure the viscosity of helium II by measuring the rate of flow through a tube were first made by Kapitza* and by Allen and Misener in the Royal Society Mond Laboratory at Cambridge. On account of the anticipated low viscosity, the latter used very narrow tubes to lessen the rate of flow, with the extraordinary result that the rate of flow was far in excess of what would occur in a liquid of viscosity $10^{-5}$ c.G.S. units. Moreover, the velocity of flow did not vary linearly with the pressure head. Kapitza found a velocity proportional to the square root of the pressure head, and interpreted this as evidence that the flow was turbulent. He gave a value of $10^{-9}$ c.G.S. units as an upper limit to the viscosity of helium II. Giauque in California has also observed the flow of helium II through an annular tube and has obtained a temperature variation of viscosity of from $10^{-6}$ to $10^{-8}$ c.G.S. units. Allen and Misener endeavoured to reduce the velocity of flow by using finer capillaries to obtain more nearly the condition for stream-line flow, and found that the rate of flow varied as a power of the pressure head of even less than one half. It was found that the dependence of velocity on the pressure head decreased with decreasing capillary size. For glass capillaries of $0.0015 \mathrm{~cm}$. radius, it was found that the velocity varied as the $1 / 6$ power of the pressure, whilst for capillaries of $5 \times 10^{-5} \mathrm{~cm}$. in radius, obtained by packing a metal tube with parallel wires and then drawing the tube through dies, the velocity became absolutely independent of the driving pressure. In the latter case, the velocity increased very rapidly with decreasing temperature and reached a value of $20 \mathrm{~cm}$. per second at $1 \cdot 1^{\circ} \mathrm{K}$. The flow also appears to be non-classical in the case of the variation of length of the capillary, since a variation of length by a factor of 78 produced only a fourfold change in the velocity.

On the other hand, experiments made by Burton in Toronto showed that with relatively wide and short tubes and rapid flow, the velocity is linearly proportional to the pressure head, and that the viscosity so determined agrees with the value obtained by the damping of oscillating disks. The anomalous features appear, therefore, when the flow takes place through very long and fine capillaries (radii less than $10^{-3} \mathrm{~cm}$.).

* Actually Kapitza used the essentially similar method of radial flow between two parallel plates with small separation. 
A little light is thrown on these curious results by the experiments of Mendelssohn and Daunt on the creep of liquid helium II in the form of mobile films over solid surfaces. If an open vessel be partially immersed in liquid helium II, it was observed that liquid gradually collected in the vessel until the liquid levels in vessel and bath were coincident. The rate of filling increased rapidly with lowering temperature. The mechanism was found to consist not in evaporation and recondensation, but in the transfer of liquid by means of surface films. At all temperatures in helium II the rate of transfer of liquid by means of the surface films was found to be independent of the difference in level. Both the thickness and the velocity of propagation of a film have been measured. The thickness is of order $5 \times 10^{-6} \mathrm{~cm}$. and the velocity increases from zero at the $\lambda$-point to $20 \mathrm{~cm}$. per second at $1^{\circ} \mathrm{K}$., which gives a rate of transfer of about $10^{-4}$ cubic centimetres per second per centimetre width of film. If films are formed above the $\lambda$-point, that is, by helium I, they are not more than $10^{-7} \mathrm{~cm}$. thick.

A rough synthesis of the experimental results on flow of helium II can be attempted as follows. In flow through a tube, two distinct but by no means separate processes are taking place*: $(a)$ the normal flow of a fluid with a viscosity (of order $10^{-4}$ c.G.S. units) which increases with decreasing temperature, and $(b)$ creep along the inside walls of the tube by means of a surface film (of thickness of the order of $5 \times 10^{-6} \mathrm{~cm}$.), the velocity of which increases rapidly with decreasing temperature. For wide tubes, effect $(a)$ predominates and the flow approximates to that of a normal viscous fluid. As the size of the capillary decreases, effect (b) becomes more pronounced, whilst for capillaries less than $10^{-4} \mathrm{~cm}$. in radius, effect $(a)$ becomes negligible and the quantity of liquid flowing per second is directly proportional to the circumference of the capillary.

Heat Conduction. The first experiments in the transport of heat through liquid helium II were made by Rollin and Keesom; their observations showed that helium II was a most efficient agent for the transport of heat, being far more effective than copper at the same temperature. It was observed later by Allen, Peierls and Uddin, and shown more convincingly by Keesom and Saris, that the rate of heat transport was not proportional to the temperature gradient. It is thus impossible to measure a true thermal conductivity for the liquid. The rate of heat transport is greater the smaller the gradient, and reaches a value corresponding to a conductivity several thousand times as great as that of copper at room temperature with gradients of the order of $10^{-5}$ of a degree per $\mathrm{cm}$.
For a given gradient the 'conductivity' increases rapidly below the $\lambda$-point to a maximum at $2 \cdot 0^{\circ} \mathrm{K}$. and then falls again. Simon and Pickard of Oxford have found that the anomalously high conductivity disappears at the temperature at which the specific heat becomes normal.

Fountain Effect. The fountain effect, which was discovered in the Mond Laboratory, shows in the most striking manner the fundamental difference between helium II and any other known liquid. In its simplest form, the effect may be described as follows. A tube is partly immersed in helium II ; the lower end of the tube is a capillary ; both ends are open and an arrangement is made to heat the liquid in the upper part. A steady flow of heat is thereby maintained down the capillary. Under these conditions, it is observed that the liquid inside the tube rises above the level outside, showing the existence of a pressure in the reverse direction to the heat flow. A more spectacular demonstration can be given by placing powder in the lower half of the immersed tube through which the heat flows. To produce the heat current in this case, it is sufficient to shine light on the powder. With this arrangement the liquid may be made to rise right out of the tube, and in fact a steady 'fountain' several centimetres in height can easily be produced.

Quantitative measurements on the magnitude of the reaction pressure are very incomplete. Some data, however, are available from measurements* on the reaction to heat flowing in helium II through a tube filled with powder particles. The reaction pressure was found to attain a maximum value of approximately half an atmosphere for a gradient of $1^{\circ}$ per $\mathrm{cm}$. at $1 \cdot 7^{\circ} \mathrm{K}$. The value of the heat conductivity through the powder-filled tube was lower by a factor of a hundred than that through a smooth capillary of the same open cross-section. It seems, therefore, that the very large conductivity observed for helium II when the heat flows through smooth tubes is caused by violent convection currents which are set up by the reaction mechanism. This might be the reason for the apparent variation of conductivity with temperature gradient.

A complete understanding of the fountain effect must naturally await a satisfactory theory of the constitution of liquid helium II. However, a few interesting deductions can be made. In the first place, in the simple arrangement described above, the force holding the liquid above the bath level can only come from some form of interaction of the liquid with the walls of the tube or the attached heating wire. No other support is available, and the vapour pressure above the bath and inside the tube are sensibly the same. Secondly, this

*Proc. Cam. Phil. Soc. (in the Press). 
interaction must result in a downward thrust on the tube equal to the weight of liquid above the bath level. From the atomic point of view, this means that the interacting atoms are steadily transferring momentum in a downward direction to the tube, just as the thrust on the walls of a vessel containing gas implies that the atoms steadily lose momentum to the walls as they are reflected. This has the interesting consequence that the main heat transport in helium II at these temperatures (below $2 \cdot 19^{\circ} \mathrm{K}$.) cannot be due to the propagation of elastic waves as in ordinary liquids and solids, since elastic waves do not carry momentum.

Many and varied hypotheses have been made concerning the constitution of helium II. Michels, Bijl and de Boer and the present authors independently suggested that certain atoms which have more than the average energy in helium II have, as well, a larger than average mean free path inside the liquid, and that heat flow represents a drift of these moving or 'excited' atoms. Later, this idea of energetic particles moving through the unexcited or 'condensed' atoms was developed by Tisza to include a theory of flow. This theory has not yet reached a quantitative stage, but has proved interesting and suggestive.

Note added in proof: Since this article was written, F. London has published (Phys. Rev., 54, 947 ; 1938) an enlargement of the theory which he based on the consideration that helium at low temperatures exhibited Bose-Einstein condensation phenomena. His theoretical interpretation of the behaviour of liquid helium II appears to be quite in accordance with the experimental deductions given above, particularly with regard to the properties of flow of both heat and liquid.

\section{The Earthquake in Chile}

$\mathrm{T}$ HE recent earthquake in Chile (Nature, Feb. 4, p. 197) may justly be regarded as one of the greatest of such catastrophes in that country, and also in the world. According to local reports by survivors, the shock started about 11.35 p.m. local time on the night of January 24, and during the two or three minutes which it lasted ruined four towns and several villages, whilst doing severe damage to several others. The earthquake was felt from Valparaiso in the north to Puerto Montt in the south, the area affected now being estimated at 300,000 square miles. Large-scale damage was done from Talca in the north to Valdivia in the south, the area chiefly concerned being the province of Nuble, and the damage to property is estimated at $£ 1,500,000$. Altogether approximately 30,000 people were killed, 50,000 injured and there are about 100,000 homeless refugees.

At Chillan, a town of 45,000 inhabitants forty miles east of Concepcion, in the neighbourhood of the epicentre, two walls of the massive cathedral, the post office and telegraph building, the O'Higgins Infantry Barracks and most of the houses collapsed immediately. A theatre and a kinema collapsed, killing all the people inside. The hospital, although badly cracked, was one of the three or four buildings left standing after the initial shocks; but owing to the number of casualties the supply of anæsthetics ran out and the surgeons were obliged to operate without. Immediately after the shock the supply of electric power and water ceased, so that the surviving firemen with the one remaining engine were powerless against the fires which followed. Moreover, transport was impossible on the railway and difficult on the roads around. After rescue operations, the Government ordered the evacuation of the town, it being estimated that here 10,000 lost their lives.

Parral, San Carlos and Egun, within the isoseismal 10, were also razed and evacuated. The villages of Retiro, Copihue, Zongavi, Bulnes and Quirihue were completely demolished. Andotros was very severely damaged, San Rosendo had 2,000 dead, and at Cauquennes, the town celebrated for its mineral springs, half the property including the hospital and prison was destroyed, killing 4,000 and injuring 800 . At Concepcion, the fourth largest city in Chile, situated near the mouth of the Bio-Bio River and having a population of 89,000 , three fifths of the city is in ruins and there are 2,000 dead. Many famous buildings, including the Teatro Campo Club and hospital, were destroyed. Here also a kinema collapsed killing 300 people inside it at the time, and fires added to the destruction in the city. It is expected that some of the fine modern buildings such as the Banco Central, the University and the Plaza Hotel which have been built to withstand earthquake shocks will have survived, unlike on the fifth occasion when Concepcion was completely destroyed on February 20, 1835. The damage was severe, but not so great at Los Angeles, Longavi and Linares. At Talcahuano, the port 